INRA Prod. Anim., 2010, 23 (5), 379-390

\title{
La filière avicole française à l'horizon 2025. Une prospective INRA-ITAVI
}

\author{
C. JEZI, C. BEAUMONT2, P. MAGDELAINE 3 \\ ${ }^{1}$ INRA, UAR1241 Expertise collective, Prospective et Etudes, F-75338 Paris, France \\ 2 INRA, UR83 Recherches Avicoles, F-37380 Nouzilly, France \\ ${ }^{3}$ ITAVI, Service Economie, 4 rue de la Bienfaisance, F-75008 Paris, France \\ Courriel : christine.jez@paris.inra.fr
}

Confrontée à une concurrence accrue dans un contexte de consommation qui se stabilise, la production française de viande de volaille s'est effondrée de $20 \%$ en 10 ans. L'exploration du futur à travers une prospective fondée sur la construction de scénarios à l'horizon 2025, met en évidence les enjeux d'avenir et les marges de manœuvre des acteurs de la filière avicole française pour favoriser ou éviter certaines évolutions.

$\mathrm{Au}$ niveau mondial et français, la viande de volaille représente $30 \%$ de la production totale de viande, se plaçant ainsi au second rang derrière la viande de porc. Elle est la troisième viande consommée en France, avec 28\% des volumes totaux consommés ; sa consommation pourrait bientôt dépasser celle de la viande bovine. Malgré cette position avantageuse, la production avicole française accuse un recul de $20 \%$ depuis la fin des années 90. Ainsi, alors que la production mondiale progresse de 3,8\% par an en moyenne sur dix ans (de 1998 à 2008), le volume de production français s'est réduit, chaque année sur la même période, de 2,3\%. Ce repli est largement lié, dans un contexte mondialisé caractérisé par une libéralisation des échanges internationaux, à une perte de compétitivité de l'aviculture française, y compris par rapport à certains partenaires européens (Magdelaine 2008). Les importations françaises ont ainsi augmenté de $150 \%$ entre 1998 et 2008 , un développement favorisé par l'essor des produits élaborés et produits de découpe, qui valorisent peu l'origine de la matière première auprès du consommateur.

Alertes sanitaires, émergence de préoccupations relatives à la durabilité des systèmes de production et au bien-être des animaux accompagnent ces évolutions, dans un contexte de libéralisation progressive des échanges de produits agricoles. Les incertitudes sur ces évo- lutions à plus long terme ont conduit l'INRA et l'ITAVI à engager une réflexion sur les perspectives d'évolution de la filière «volailles de chair» en France ${ }^{1}$.

L'idée d'associer les compétences et réseaux professionnels des deux instituts s'est concrétisée début 2008 par la mise en place d'une prospective dont l'objectif est de fournir des éléments de réflexion aux décideurs impliqués dans l'orientation de la recherche et les politiques publiques, et plus spécifiquement, dans la définition de stratégies collectives pour la filière française. Cet article en présente les principaux résultats et enseignements.

\section{1 / Méthodologie : l'explo- ration des futurs possibles grâce à des scénarios}

Les évolutions passées et en cours nous enseignent que le seul suivi des tendances du marché ne suffit pas à anticiper l'avenir. Il est également nécessaire d'identifier les incertitudes majeures et les risques ou opportunités de ruptures en considérant les transformations du contexte géopolitique, de la société, de la technologie et des stratégies d'entreprises. Mais les incertitudes pesant sur ces évolutions rendent difficile la prévision et imposent la prise en compte de différents futurs possibles pour chaque facteur «moteur». C'est pourquoi il a été choisi d'explorer le futur en construisant des scénarios, selon une méthode de prospective dite d'analyse morphologique. Cette démarche ne vise pas à prédire l'avenir, mais à l'explorer à travers différentes visions, pour se préparer à différents changements possibles, voire à en être l'initiateur. Ainsi, la construction de scénarios permet d'alerter les acteurs et les décideurs sur des phénomènes ou des enjeux encore en germe afin d'anticiper leurs conséquences ou de construire des stratégies proactives (De Jouvenel 2004).

L'opération s'est appuyée sur une équipe projet INRA-ITAVI, qui a animé et alimenté les réflexions d'un groupe de travail chargé de construire des scénarios d'avenir. Ce groupe a réuni à une dizaine de reprises sur 18 mois (2008-2009) des chercheurs, des acteurs de la filière et de la société civile, qui ont choisi collectivement de se projeter à l'horizon d'une quinzaine d'années (2025) pour considérer une grande variété de facteurs d'évolution et de possibilités de rupture tout en restant pertinent pour les décideurs. En complément, ont été réalisées des auditions de personnalités dont les secteurs d'activité, les fonctions ou les sensibilités n'étaient pas représentées dans le groupe de travail. Cette approche collective a permis de croiser un large éventail de connaissances, d'expériences et de regards.

\footnotetext{
${ }^{1}$ La filière «volailles de chair» est définie comme l'ensemble des systèmes d'acteurs directement impliqués à tous les stades depuis l'élevage des poussins jusqu'au produit transformé.
} 
La première étape de la construction de scénarios a consisté à identifier les facteurs de toute nature qui exercent ou sont susceptibles d'exercer une influence sur la filière avicole française à l'horizon 2025, et de les regrouper par grands thèmes. Ensuite, les évolutions passées et en cours ont été analysées afin de définir des tendances, lourdes ou émergentes, et les ruptures possibles. Le groupe de travail a choisi alors des hypothèses d'évolution vraisemblables, pour chaque facteur à l'horizon 2025 . Combinées entre elles, ces hypothèses constituent des scénarios. Parmi les multiples combinaisons possibles, le choix des scénarios s'est fondé sur les critères suivants :

- ils sont pertinents pour l'action, en ce qu'ils soulignent bien les enjeux, les incertitudes, les risques et les opportunités que la réflexion stratégique doit considérer,

- ils sont cohérents, c'est-à-dire qu'ils proposent des combinaisons logiques d'hypothèses pour donner une représentation cohérente de l'ensemble du système,

- ils sont vraisemblables,

- ils sont transparents, c'est-à-dire lisibles et justifiés,

- ils sont suffisamment contrastés pour offrir une large vision des futurs possibles.

L'objectif était de construire des scénarios de la filière avicole française, mais la réflexion s'est située sur un terrain beaucoup plus large en s'ouvrant aux évolutions des contextes européens et mondiaux, dont certaines seront déterminantes pour le devenir de la filière française.

Un travail de quantification de quelques indicateurs à l'horizon 2025, à dires d'experts, a ensuite permis de vérifier la cohérence de chacun des scénarios et de leur donner une illustration plus concrète. Enfin, ont été mis en évidence les enjeux pour le futur et les leviers d'action pour les acteurs de la filière, les politiques et la recherche.

\section{2 / Etat des lieux de la filiè- re avicole française}

\section{1 / Les grandes étapes du déve- loppement de l'aviculture fran- çaise depuis 1950}

L'analyse historique du développement de l'aviculture française depuis le début des années 50 peut se décomposer en quatre grandes périodes :

1/ Les années 60 ou "années d'apprentissage». Elles se caractérisent par l'émergence d'une aviculture intégrant des innovations techniques importantes (en génétique, nutrition et santé animale), dans un contexte de demande alimentaire soutenue et de mise en place du marché européen, principaux moteurs du développement sur cette période. Dès cette époque, une spécialisation régionale et l'organisation de partenariats forts entre producteurs et industriels se mettent en place.

2/ Les années 70 et le début des années 80. L'expansion se poursuit dans un contexte politique favorable aux exportations de l'Union Européenne vers les pays tiers. Dans le même temps, la concentration régionale s'accentue, plus particulièrement en Bretagne, où les élevages s'orientent vers la production de poulet pour le grand export et la production de dinde. Dans les autres régions, l'aviculture reste principalement tournée vers le marché national. C'est dans ce contexte que la production fermière se structure autour du Label Rouge.

3/ Les années 85-95. Elles correspondent à la consolidation de la position française en Europe ; la croissance est alors alimentée par le développement simultané de la consommation nationale et du marché communautaire. Les filières bénéficient encore de subventions directes aux exportations et de protections vis-à-vis de la concurrence internationale grâce à des droits de douanes dissuasifs sur les produits importés.

4/ De 1995 à aujourd'hui : c'est la fin de «l'âge d'or». Suite à la signature des accords de Marrakech en 1994, les soutiens aux exportations et les droits de douanes sont réduits. Ces mesures sont déterminantes pour les échanges fran- çais de volailles avec les pays tiers, du fait du différentiel de coût de production entre la France et ses nouveaux compétiteurs sur le marché international. La consommation intérieure, jusque là très dynamique, se stabilise. La période est, par ailleurs, marquée par différentes crises sanitaires qui alimentent la méfiance des consommateurs vis-à-vis d'une agriculture perçue par certains comme étant industrielle et polluante (figure 1).

Le repli de la production française a pour effet une diminution des marges de chacun des maillons de la filière et un ralentissement des investissements, générant un vieillissement des outils et une aggravation du déficit de compétitivité de la filière.

\section{2 / Une consommation indivi- duelle qui se stabilise}

Le niveau de la consommation individuelle de viandes, qui avait pourtant connu un développement régulier en France jusqu'au début des années 80 (Combris 1997), tend globalement à stagner autour de $90 \mathrm{~kg}$ par personne et par an pour les principales viandes. $\mathrm{La}$ croissance de la consommation de viande de volaille s'est quant à elle poursuivie jusqu'en 2001 où elle a atteint un maximum de $26 \mathrm{~kg}$ par personne et par an. La part des volailles dans la consommation globale de viandes est ainsi passée de $15 \%$ au début des années 70 à $28 \%$ en 2008 (Agreste conjoncture 2008). L'originalité de la consommation française réside dans la diversité des espèces et la place relativement modeste du poulet (58\% en 2008). Cependant, sur la dernière décennie, la consommation de poulet progresse, ainsi que celle de canard, alors que celles de dinde et

Figure 1. Production, consommation, et taux d'autoapprovisionnement (Production/ Consommation en \%) des filières françaises en viande de volaille depuis 1970 (ITAVI d'après SSP).

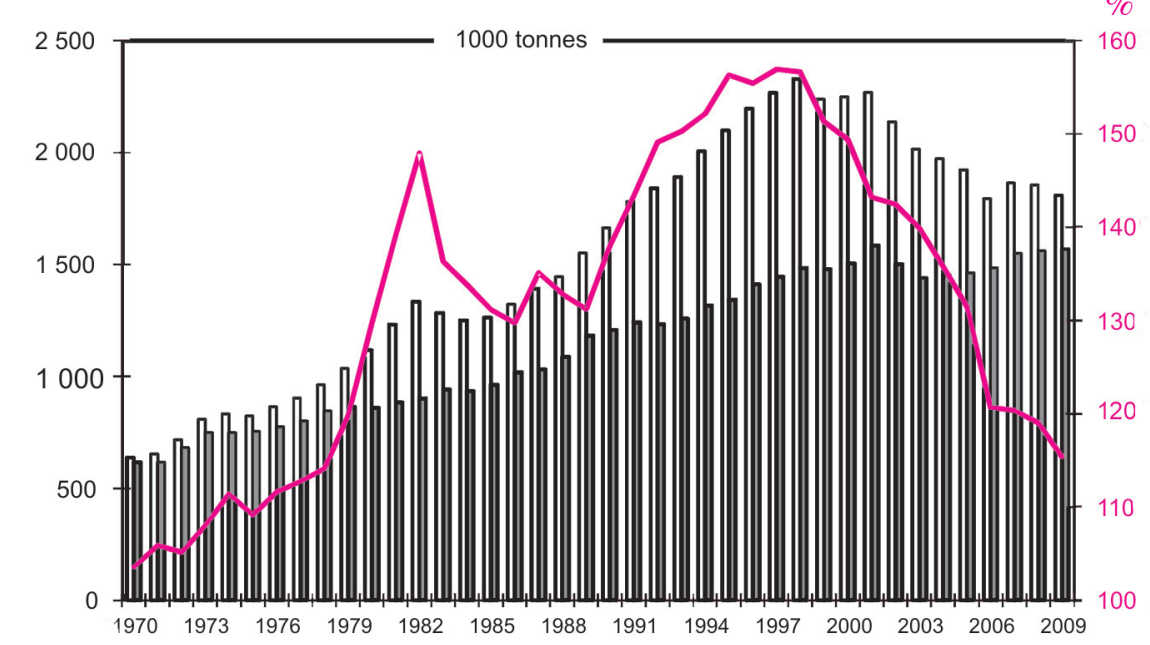


Figure 2. Evolution de la consommation individuelle de viandes par espèce (Office de l'élevage).

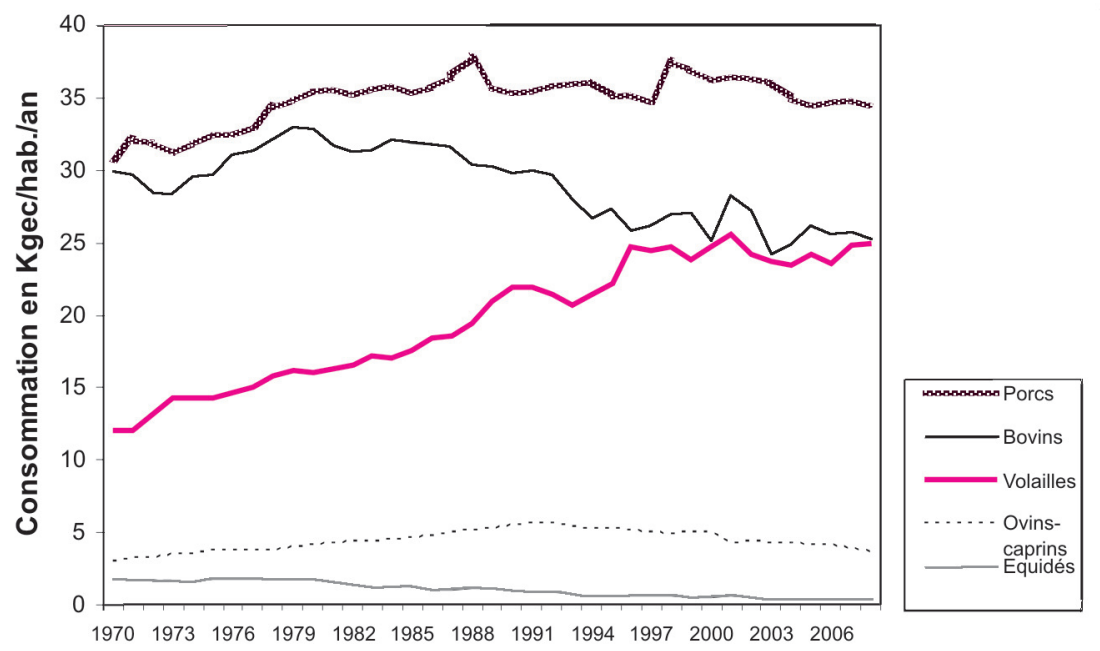

de pintade régressent (Magdelaine 2008).

La stagnation de la demande individuelle de viande de volaille s'accompagne d'une forte segmentation des marchés, faisant une part croissante aux produits élaborés, au détriment des volailles entières et des produits moins transformés. Ainsi, le poulet entier ne représente plus que $38 \%$ des achats des ménages de viande de poulet, contre $40 \%$ pour les produits de découpes et $22 \%$ pour les produits élaborés. Ces derniers, ne favorisant pas la valorisation d'une origine ou d'un mode de production spécifiques, accentuent la vulnérabilité de la production française vis-à-vis de la concurrence étrangère (figure 3).

Figure 3. Répartition des achats des ménages en poulet (ITAVI d'après TNS).

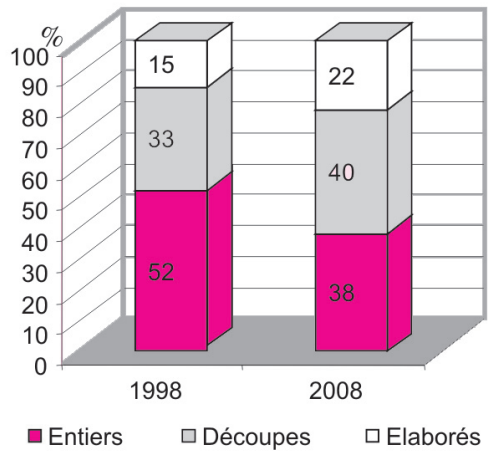

Ces évolutions de marchés et de modes de consommation sont peu favorables aux filières respectant un cahier des charges Label Rouge, Certification de Conformité Produit (CCP) ou Bio, qui représentent cependant près du quart de la production de volailles. Ainsi, si le Label Rouge constitue encore aujourd'hui un segment essentiel et moteur du marché des volailles entières, il peine en revanche, à se développer sur le marché des produits de découpe et transformés (Magdelaine 2008).

\section{3 / Des élevages encore peu spécialisés}

En 2000, lors du dernier recensement agricole, environ 20000 élevages, employant 14000 équivalents temps plein, assuraient l'essentiel de la production nationale de volailles de chair. La surface agricole utile des exploitations était de 51 hectares, une moyenne entre des élevages spécialisés de très faible surface $(23 \%$ des exploitations disposant de moins de 10 ha) et des structures agricoles plus diversifiées, installées sur de plus importantes superficies (Agreste Cahiers 2001). L'aviculture française est en effet peu spécialisée; en 2004, seulement 30\% des producteurs avaient un revenu avicole représentant plus de $75 \%$ de leur revenu agricole total. Ces éleveurs représentaient $40 \%$ des capacités de production en volailles de chair. Le plus souvent en effet, l'aviculture se développe aux côtés d'un atelier de bovins lait ou viande, ou de grandes cultures (Agreste Chiffres et données 2006).

Les relations entre les éleveurs et les industriels sont définies par des contrats dits «d'intégration» fondés sur la gestion des plannings de production par l'aval, et une rémunération des éleveurs peu liée au contexte de marché de la viande de volaille. Dans la plupart des cas, l'intégrateur fournit les poussins, les aliments, les médicaments, apporte le conseil technique et le support vétérinaire, assure la livraison des aliments et collecte les animaux finis selon un planning qu'il a lui-même défini. L'éleveur de son côté fournit le capital investi dans les bâtiments et les équipements d'élevage, le travail de gestion de l'élevage au quotidien, ainsi que l'électricité, le gaz et l'eau nécessaires à la production (Lavergne 2003). Ce fonctionnement a facilité l'adéquation de la réponse des industriels aux besoins des consommateurs. Cependant, la contraction des débouchés et la pression concurrentielle ont fait apparaitre certaines limites. En effet, les contrats à façon ne font que garantir à court terme un niveau de marge objectif pour un niveau de performances moyen. Certains acteurs de la filière s'interrogent sur leur efficacité pour optimiser les performances techniques en élevage, et celle plus globale de la filière. Ils ne permettent pas non plus de sensibiliser les éleveurs aux évolutions du marché. Ils n'ont par ailleurs aucunement protégé les éleveurs face à la réduction d'activité ; ainsi, les producteurs les plus sensibles à la concurrence internationale n'ont pu, compte tenu de la baisse de leur chiffre d'affaires, réaliser les investissements nécessaires à la rénovation et à la modernisation des bâtiments. Par conséquent, si l'intérêt de la contractualisation semble partagé par tous compte tenu de la souplesse et de l'efficacité que ce système apporte aux industriels en termes de gestion de volumes d'approvisionnement, d'investissement et d'adéquation qualitative de l'offre à la demande, des évolutions semblent nécessaires afin d'optimiser la compétitivité globale de la filière.

\section{4 / Un secteur industriel qui se restructure}

En France, le secteur de l'aviculture «chair» est organisé autour de quelques grands groupes qui intègrent plus ou moins complètement l'amont (aliments et poussins) et l'aval des filières (abattage et transformation). La restructuration qui a abouti à leur création s'est déroulée progressivement, depuis la fin des années 80, au gré des opportunités de reprise et des crises. Elle s'accompagne d'une évolution "capitalistique» de l'industrie avicole (automatisation de plus en plus marquée et poussée des process, degré croissant d'élaboration des produits, couplés à un renforcement des investissements nécessaires à la mise aux normes sanitaires) qui tend à exclure certaines PME familiales (Magdelaine 2008).

Cependant, l'industrie avicole française est moins concentrée que d'autres industries agroalimentaires en France, comme l'industrie laitière par exemple, et que d'autres industries avicoles en Europe comme celles de l'Allemagne ou de l'Italie. En effet, le tissu industriel reste caractérisé par la présence, aux 
Figure 4. Evolution des exportations françaises de viandes et préparations de volailles depuis 1997 (Douanes, Agreste).

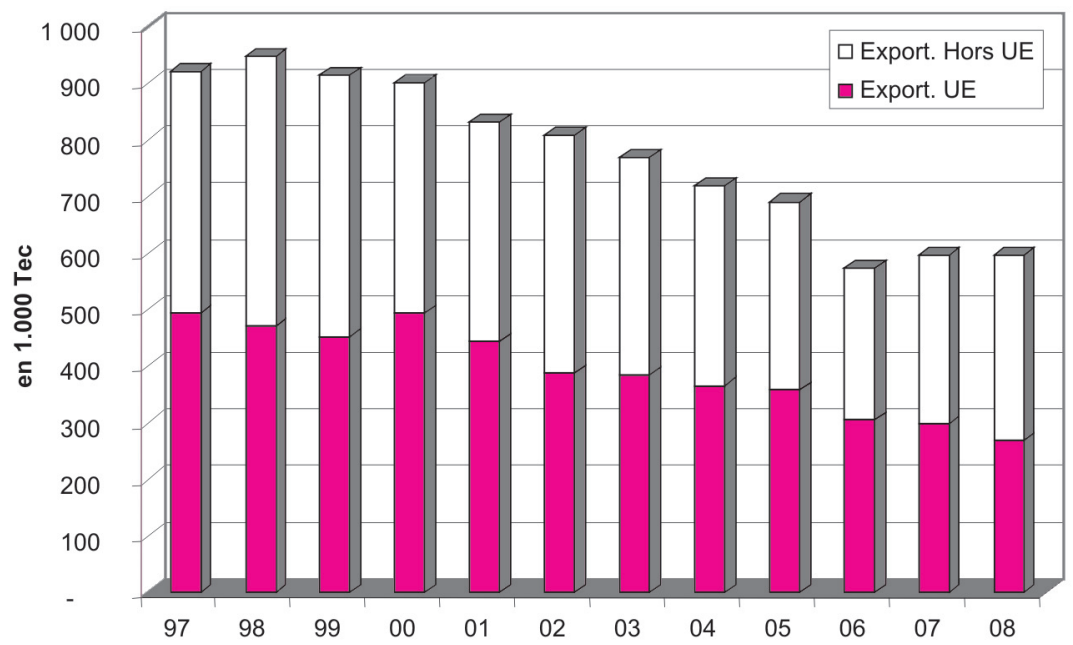

côtés de ses leaders, de nombreuses petites et moyennes entreprises. Ainsi, en 2007, les quatre premiers groupes français assuraient $63 \%$ du chiffre d'affaires de la filière volailles de chair (produites en France), les dix premiers $80 \%$ (And-I, 2008). La perte de marchés européens et le développement de la concurrence internationale ont accéléré la dynamique de restructuration et de concentration, dont les prochaines étapes se joueront probablement à l'échelle européenne.

\section{5 / Une position affaiblie sur le marché international}

En 1998, la France exportait plus de $40 \%$ de sa production de volailles, dont la moitié vers des pays de l'Union Européenne. Cette vocation exportatrice s'est considérablement affaiblie à la suite de la signature des accords en 1994 de Marrakech qui ont provoqué la chute des ventes hors Europe. De plus, depuis 1998, la concurrence des produits congelés importés des pays tiers s'est combinée au développement des productions nationales, notamment allemandes et polonaises, pour induire un recul des exportations françaises sur le marché européen (figure 4).

Les différentiels de compétitivité sur le prix entre la filière avicole européenne et ses principaux compétiteurs sur le marché international subsistent. En 2006, l'écart de coût de production (hors rémunération de l'éleveur) entre la France et le Brésil était en effet estimé à $0,24 € / \mathrm{kg}$ vif, soit $35 \%$ du coût français (figure 5).

En 2006, de nouveaux accords ont été conclus par l'Union Européenne, l'un avec le Brésil et l'autre avec la

\section{6 / Un enjeu fort : le maintien de l'activité de production}

Dans un marché mondial en développement et fortement concurrentiel, l'enjeu majeur pour les filières avicoles française et européenne est de conserver la maîtrise de l'approvisionnement du marché national, et en partie communautaire, malgré des handicaps de coût de production par rapport à des concurrents brésiliens, nord-américains ou asiatiques. Ce défi se double pour la filière avicole française de celui de l'amélioration de sa compétitivité sur le marché intra-européen. Le maintien de l'activité est important pour les 60000 emplois directs générés par la filière, mais aussi pour l'équilibre d'autres secteurs économiques de nombreuses régions. Entre autres, l'aviculture «chair» est un débouché important des filières de grandes cultures et elle apporte un complément de revenu à de nombreuses exploitations de polyculture-élevage, contribuant à leur survie.

Thailande. Ils accordent à ces pays contingents d'importation à droits réduits pour les viandes salées, les préparations de dinde et les préparations cuites de poulet, ce qui génère une nouvelle augmentation des importations européennes (Trégaro et Vallin 2009). Cette pression accrue sur le marché communautaire, conjuguée à une perte de compétitivité intra-communautaire des industriels français sur les produits frais, pénalise fortement les ventes françaises et le solde des échanges entre la France et ses partenaires européens. Il est maintenant structurellement déficitaire.

\section{3 / L'avenir de la filière avi- cole française : trois com- posantes d'évolution ma- jeures}

A partir des dynamiques passées d'évolution et des questionnements actuels sur l'avenir de la filière avicole française, une quarantaine de facteurs influençant l'évolution de la filière avicole ont été mis en évidence. Ils ont été hiérarchisés et répartis en trois

Figure 5. Répartition des coûts de production sortie (élevage hors maind'œuvre éleveur) en France et au Brésil (Etat de Santa Catarina) en 2006 (ITAVI et Céleres).

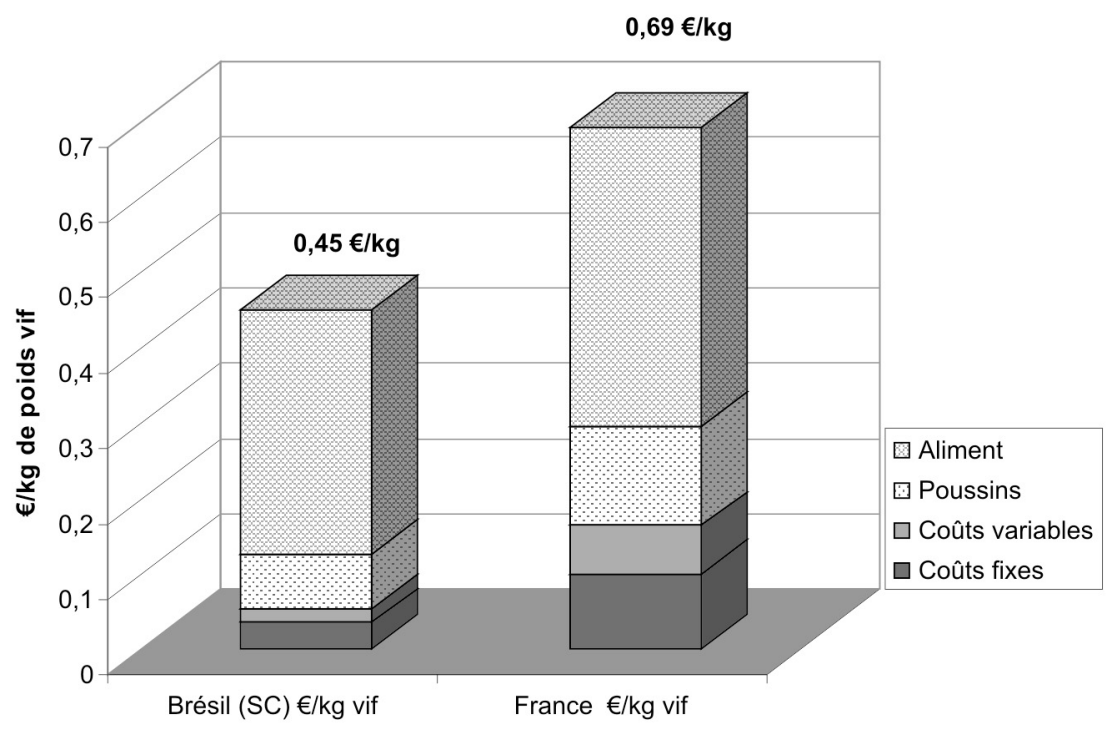


composantes majeures qui constituent la base de la construction des scénarios : 1) la consommation, le comportement des consommateurs et des citoyens, 2) les politiques publiques internationale, communautaire et française, et 3) les stratégies des acteurs, notamment de la transformation, et la structuration des filières.

\section{1 / La consommation : vers une nouvelle segmentation des mar- chés?}

Depuis le début des années 90, la consommation globale de viande tend à stagner en France comme dans les principaux pays de l'Union Européenne, alors que la part de la viande de volaille dans la consommation de viande est en légère augmentation grâce à ses atouts économiques, à la bonne image des viandes blanches, à son aptitude à la transformation et à la résistance aux crises sanitaires (Magdelaine et al 2008). Cette évolution s'accompagne d'un développement de la consommation de produits élaborés qui favorise les importations au détriment de la production domestique. Les tendances lourdes évoquées peuvent cependant subir des inflexions. Ainsi, un contexte de crise économique pourrait favoriser l'achat de produits bruts moins onéreux ; à l'inverse, le renforcement de critères d'achat autres que le seul prix (impact environnemental, bien-être animal, préférence locale...) pourrait renforcer l'attrait des produits domestiques dans un contexte de pouvoir d'achat plus favorable (Jez et al 2009).

\section{2 / Les politiques publiques internationale, communautaire et française : de grandes incerti- tudes}

Compte tenu des conséquences de l'accord de Marrakech sur les échanges français de viande de volaille, les politiques publiques et les régulations internationales qui régissent la production et le commerce international ont été considérées comme les plus déterminantes pour l'avenir de la fillière avicole. Elles ont, en effet, un impact direct sur la compétitivité-prix de l'aviculture européenne et donc sur sa capacité à maintenir ou développer ses parts de marchés. Mais les difficultés des négociations agricoles à l'Organisation Mondiale du Commerce (OMC) dans le cadre du cycle de Doha rendent très difficile l'anticipation des réglementations internationales, multilatérales et bilatérales, qui gouverneront les échanges de biens agricoles et agroalimentaires en 2025 (Tregaro et Vallin 2009). Il en est de même des politiques et réglementations européennes et, notamment, de la poli- tique agricole, qui jusqu'alors concernait peu l'aviculture en dehors des mesures de soutien à l'exportation. Les incertitudes sur leur évolution permettent d'envisager différents développements et ruptures à l'horizon 2025.

\section{3 / Les stratégies industrielles : concentration et forte concur- rence extracommunautaire}

L'observation des évolutions des dix dernières années montre que, pour faire face au développement de la concurrence, l'effort de la filière a principalement porté sur la réduction des coûts unitaires de production. Cette rationalisation des coûts a limité les capacités financières à investir pour d'autres ambitions et a conduit à une concentration de la production et de la transformation, fragilisant certaines filières régionales.

Ainsi, en France, mais aussi dans les pays européens voisins, l'industrie avicole se concentre autour de groupes leaders atteignant jusqu'à 35 à $50 \%$ de part de marché dans leur pays d'origine. Ces groupes demeurent le plus souvent nationaux, même si certains développent leur activité à l'international (OECD 2006). Il n'existe pas aujourd'hui de groupe européen transnational en aviculture, alors que se constituent et se renforcent, dans d'autres pays (Brésil, Thaïlande, Etats-Unis...), de grands groupes mondiaux de la viande. Aujourd'hui, la possibilité d'une prise de contrôle de la transformation hexagonale par de puissants groupes d'autres continents fait peser de lourdes incertitudes sur les stratégies d'approvisionnement des futurs leaders de l'industrie avicole (Trégaro et Vallin 2009). Face à ces concurrents, qui dominent en compétitivité-prix, les marges de manœuvre sont limitées. Cependant, des opportunités semblent exister en faveur de la production locale sous réserve de développements de ses débouchés et de sa rentabilité (Jez et al 2009).

\section{4 / 2025 : quatre scénarios pour la filière avicole fran- çaise}

A partir de différentes hypothèses sur l'évolution des trois composantes citées précédemment, quatre scénarios ont été construits. Deux d'entre eux accentuent des tendances déjà à l'œuvre. Le scénario 1 «La chair de poule : la filière avicole happée par la mondialisation» envisage le futur de la filière avicole dans un contexte où le marché des produits agricoles est de plus en plus libéralisé ; le scénario 3 «La poule est dans le pré : le développement durable anime la filière» se caractérise par la prise en compte centrale de l'environnement dans le comportement des citoyens et des consommateurs français et européens. Les deux autres scénarios sont davantage en rupture. Dans le scénario 2 «Le coq européen : l'aviculture française, un pilier de l'industrie avicole européenne», la rupture tient à un changement de cap dans la construction européenne, qui serait davantage axée sur la protection et la création d'emplois. Le scénario 4 «La poule au pot : la filière avicole française dans une régulation alimentaire mondiale» envisage un contexte de crises alimentaires répétées, qui feraient émerger une régulation mondiale du commerce de produits alimentaires, dans un objectif de sécurité alimentaire des populations et de protection des ressources naturelles (figure 6).

Scénario 1 - La chair de poule : la filière avicole happée par la mondialisation

En 2025, le marché européen est totalement ouvert aux importations de produits avicoles dès lors que ceux-ci respectent les normes sanitaires internationales. L'industrie avicole, dominée par de grands groupes américains et brésiliens, s'approvisionne dans le monde entier, privilégiant les matières premières à bas prix. Son offre répond bien à la transformation des modes de vie et des habitudes alimentaires des européens. En effet, dans une société ouverte aux influences culturelles multiples, les consommateurs, dont le pouvoir d'achat progresse, privilégient les plats cuisinés, les aliments faciles et rapides à préparer et à consommer. La moitié de la viande de volaille consommée par les français est importée. Elle est commercialisée sous forme de produits élaborés dans des gammes qui se sont multipliées pour répondre à la variété des attentes de la population: recettes exotiques, produits snack ou bien festifs, labels garantissant le respect du bien-être animal ou de l'environnement ou encore le commerce équitable. Ainsi, en 25 ans, la production française de volaille a perdu près de $50 \%$ de son volume ; les élevages, concentrés dans les zones de production les plus compétitives, fournissent principalement le marché des produits frais et ultra-frais.

Scénario 2 - Le coq européen : l'aviculture française, un pilier de l'industrie avicole européenne

En 2025, le marché européen des produits avicoles reste protégé par des droits de douane, et des normes sanitaires plus exigeantes que les standards internationaux. La crise économique mondiale 2008-2015 et l'absence de reprise durable ont en effet stoppé le 
Figure 6. Représentation simplifiée des combinaisons d'hypothèses aboutissant aux scénarios.

\begin{tabular}{|c|c|c|c|c|}
\hline Facteurs d'évolution & \multicolumn{4}{|c|}{ HYPOTHESES D EVOLUTION A LHORZON 2025} \\
\hline $\begin{array}{l}\text { Politiques et } \\
\text { règlementations }\end{array}$ & $\begin{array}{l}\text { Libéralisation des } \\
\text { échanges et fin des } \\
\text { aides directes à } \\
\text { l'agrimulture }\end{array}$ & $\begin{array}{l}\text { L'emploi et la santé } \\
\text { publique au coeur des } \\
\text { politiques européennes }\end{array}$ & $\begin{array}{l}\text { Environnement et } \\
\text { qualité au centre des } \\
\text { politiques agricoles } \\
\text { dans un contexte de } \\
\text { libéralisation }\end{array}$ & $\begin{array}{l}\text { Une gouvernance } \\
\text { mondiale pour nourrir le } \\
\text { monde }\end{array}$ \\
\hline $\begin{array}{l}\text { Comportements de } \\
\text { consommation }\end{array}$ & $\begin{array}{l}\text { Une culture alimentaire } \\
\text { composte, des } \\
\text { influences multiples }\end{array}$ & $\begin{array}{c}\text { Des consommateurs à la } \\
\text { recherche des prix les } \\
\text { plus bas }\end{array}$ & $\begin{array}{l}\text { Moins de viande dans } \\
\text { l'assiette; des } \\
\text { consommateurs qui } \\
\text { priviégient la qualité }\end{array}$ & \\
\hline \multirow[t]{2}{*}{$\begin{array}{l}\text { Stratégies des acteurs et } \\
\text { structuration de la filière }\end{array}$} & 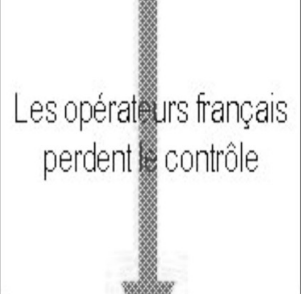 & $\begin{array}{l}\text { Une structuration des } \\
\text { filières autour de } \\
\text { quelques grands } \\
\text { opérateurs européens } \\
\text { spécialisés }\end{array}$ & $\begin{array}{l}\text { Une diversité d'acteurs } \\
\text { dans une flière } \\
\text { proactive }\end{array}$ & $\begin{array}{l}\text { De grands acteurs } \\
\text { mondiaux spécialisés } \\
\text { qui optimisentles } \\
\text { process et standardisent } \\
\text { looffe }\end{array}$ \\
\hline & $\begin{array}{l}\text { "La chair de poule " } \\
\text { La filière avicole happée } \\
\text { par la mondialisation }\end{array}$ & $\begin{array}{l}\text { "Le coq européen " } \\
\text { L'aviculture française, } \\
\text { un pilier de l'industrie } \\
\text { avicole européenne }\end{array}$ & $\begin{array}{l}\text { "La poule est } \\
\text { dans le pré " } \\
\text { Le développement } \\
\text { durable anime la filière }\end{array}$ & $\begin{array}{l}\text { "La poule au pot " } \\
\text { La filière avicole } \\
\text { française dans une } \\
\text { régulation alimentaire } \\
\text { mondiale }\end{array}$ \\
\hline
\end{tabular}

processus de libéralisation, même si de grands blocs de pays se sont renforcés, comme l'Union Européenne, ou se sont constitués autour d'accords commerciaux. Le développement de politiques industrielles coordonnées en Europe et le maintien de ses droits de douane ont largement profité à son industrie avicole qui s'est restructurée et modernisée. Dominée par de grands groupes européens transnationaux, la filière approvisionne un marché très standardisé, pour lequel le prix est le principal critère de choix des consommateurs. La tendance à la baisse de la production française de volaille, caractéristique du début du 21 ème siècle, s'est nettement ralentie et, en 2025 , le grand ouest français reste l'un des plus grands bassins de production européens de volaille.

Scénario 3 - La poule est dans le pré: le développement durable anime la filière

En 2025, l'Europe mise sur ses atouts en matière de développement durable pour faire face à la concurrence internationale et développer une production agricole de qualité sur son territoire. Elle a su tirer parti de ses compétences technologiques mais aussi d'un niveau d'exigence élevé des consommateurs en matière de préservation de l'environnement et de qualité des produits. L'alimentation est un sujet de préoccupation important des européens. Les consommateurs, qui mangent en moyenne moins de viande, privilégient les produits bruts et la qualité, celle-ci étant d'ailleurs fréquemment perçue comme associant santé, respect de l'environnement et du bien-être animal. Ces évolutions, dans un contexte de croissance mondiale forte, ont été plutôt favorables à la filière avicole européenne, qui a moins souffert de la baisse de la consommation de viande que d'autres filières. Elle a su s'inscrire dans une dynamique collective en améliorant son bilan carbone et en adaptant ses processus de production pour réduire les pollutions et les émissions de gaz à effet de serre mais aussi pour respecter des normes exigeantes en matière de bienêtre animal. La grande distribution a joué un rôle moteur dans cette évolu- tion, en relayant les attentes des consommateurs. La filière française a regagné en compétitivité en améliorant ses performances en termes de durabilité. En 2025, elle exporte des produits à haute valeur ajoutée, même si elle n'a pas retrouvé, du fait la concurrence internationale et de l'évolution des régimes alimentaires, ses volumes de production de la fin du 20 ème siècle.

Scénario 4 - La poule au pot : la filière avicole française dans une régulation alimentaire mondiale

Dans un monde profondément affecté par une succession d'accidents climatiques et de crises sanitaires, la coordination des Etats pour assurer une sécurité alimentaire mondiale durable s'est imposée. La production agricole est encouragée sur tous les continents avec des exigences fortes en matière de préservation des ressources naturelles et de qualité sanitaire. Dans ce contexte, l'Europe met son autosuffisance alimentaire au premier plan pour rassurer des citoyens marqués par la peur de manquer et focalisés sur les prix. 
Tableau 1. Les scénarios en chiffres.

\begin{tabular}{|l|c|c|c|c|c|c|}
\hline & 1998 & 2008 & \multicolumn{4}{|c|}{2025} \\
\cline { 4 - 7 } & & & $\begin{array}{c}\text { Scénario } \\
\mathbf{1}\end{array}$ & $\begin{array}{c}\text { Scénario } \\
\mathbf{2}\end{array}$ & $\begin{array}{c}\text { Scénario } \\
\mathbf{3}\end{array}$ & $\begin{array}{c}\text { Scénario } \\
\mathbf{4}\end{array}$ \\
\hline $\begin{array}{l}\text { Consommation individuelle de } \\
\text { viande de volaille (kg/hab./an) }\end{array}$ & $\mathbf{2 4 , 7}$ & $\mathbf{2 4 , 6}$ & 28 & 25 & 22 & 25 \\
\hline $\begin{array}{l}\text { Part des viandes consommées } \\
\text { sous forme de produits élaborés }\end{array}$ & $\mathbf{1 5 \%}$ & $\mathbf{2 5 \%}$ & $50 \%$ & $25 \%$ & $20 \%$ & $25 \%$ \\
\hline $\begin{array}{l}\text { Part des importations dans la } \\
\text { consommation }\end{array}$ & $10 \%$ & $\mathbf{2 4 \%}$ & $50 \%$ & $30 \%$ & $24 \%$ & $20 \%$ \\
\hline $\begin{array}{l}\text { Production brute de volailles } \\
\text { (1000 Tec) }\end{array}$ & $\mathbf{2 , 3 1}$ & $\mathbf{1 , 8 6}$ & 1,28 & 1,74 & 1,63 & 1,92 \\
\hline $\begin{array}{l}\text { Auto approvisionnement } \\
\text { (production/consommation) }\end{array}$ & $\mathbf{1 5 6 \%}$ & $\mathbf{1 1 8 \%}$ & $67 \%$ & $102 \%$ & $109 \%$ & $113 \%$ \\
\hline
\end{tabular}

Sources pour 1998 et 2008 : ITAVI, Office de l'Elevage, INSEE ; les indicateurs pour 2025 sont des hypothèses du groupe de travail.

L'industrie avicole est sous contrôle de grands groupes transnationaux implantés sur tous les continents pour sécuriser les approvisionnements. La France, malgré un contexte économique mondial difficile, affirme sa capacité à garantir la régularité, la durabilité et la sécurité sanitaire de sa production grâce à une agriculture basée sur les hautes technologies ; elle n'importe plus, en 2025 , que $20 \%$ de sa consommation de viande de volaille. Ses exportations et sa production, en baisse jusqu'en 2020 , sont de nouveau en augmentation (tableau 1, figure 7).

Aucun scénario n'envisage une reprise de la production française. Bien au contraire, l'illustration chiffrée des futurs possibles de la filière française traduit ses difficultés à maintenir les niveaux de production actuels compte tenu des évolutions possibles de la con-

Figure 7. La production totale de viande de volaille en France : évolution de 1970 à 2008, et en 2025 dans les scénarios (données 1970-2008, Office de l'élevage).

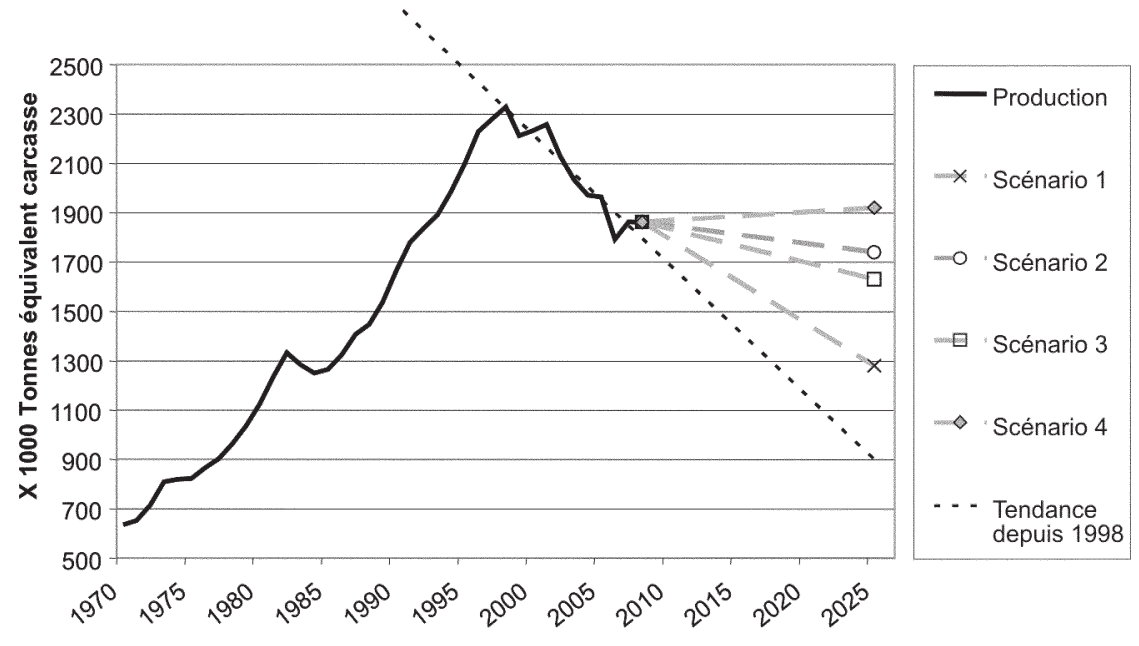

nologiques et environnementaux de la viande de volaille lui permettent de faire face à de multiples évolutions des comportements de consommation. Mais cette force, si elle garantit un certain niveau de consommation, n'est pas toujours suffisante pour maintenir une filière française, qui souffre d'un déficit de compétitivité-prix ${ }^{2}$. Aujourd'hui, par exemple, le surcoût de production entre les filières européennes et brésiliennes est de l'ordre de 50\% (Van Horne 2009). Il est difficile d'envisager qu'il se réduise significativement à. l'horizon 2025 , ce qui signifie qu'à long terme, le maintien de la production sur le territoire européen repose soit sur l'existence de mesures de protection (droits de douane ou normes de production identiques à celles imposées aux producteurs européens), soit sur la valorisation d'éléments de compétitivité hors-prix ${ }^{3}$ tels que la qualité des produits, la proximité ou la fraîcheur. C'est dans le scénario 1 que le déficit de compétitivitéprix de la filière française est le plus manifeste. En effet, d'une part, dans un contexte de libéralisation poussée des échanges, les importations sont soumises à de faibles droits de douane, et d'autre part, la consommation de produits élaborés, peu identifiés par leur origine, se développe. Le scénario 2, dans lequel l'industrie avicole européenne bénéficie de protection aux frontières vis-à-vis des pays tiers, souligne que le déficit de compétitivité-prix de la filière française existe aussi vis-àvis de ses concurrents européens. Même si le volume de production française en 2025 est plus élevé dans ce scénario que dans les scénarios 1 et 3 , il ne retrouve pas son niveau de la fin des années 90 , du fait de la concurrence intra européenne et de la diminution des exportations.

\footnotetext{
${ }^{2}$ La compétitivité-prix s'apprécie par la capacité d'une entreprise ou d'une filière à produire à moindre coût le même produit (qualité comparable) que ses concurrents.

${ }^{3}$ La compétitivité hors-prix évalue la capacité à innover en fabricant un produit différent de celui des concurrents et à valoriser cette différence auprès de ses clients.
} 
Du maintien des filières dépendent des emplois, plus particulièrement dans les zones de forte densité d'élevage. Comme le nombre d'éleveurs diminue avec la spécialisation des ateliers et les départs en retraite, l'impact social d'une potentielle baisse d'activité pourrait être atténué. Dans le scénario 1 par exemple, la diminution de l'activité entraîne une décroissance de l'emploi salarié avec le transfert des tâches telles que l'abattage vers des fonctions d'élaboration et de transformation; les difficultés de recrutement dans ces secteurs qui attirent de moins en moins de travailleurs permettent de faire face à ces évolutions sans licenciements massifs. En revanche, le redéploiement de l'activité sur le territoire, comme c'est le cas dans le scénario 3, pourrait être limité par le manque d'éleveurs, plus particulièrement dans les zones de faible tradition avicole. Rendre la profession attractive pourrait ainsi devenir un enjeu majeur. L'acceptabilité de l'élevage, le confort de travail de l'éleveur et sa capacité à générer un revenu sont des éléments clés pour y parvenir.

Enfin, les enjeux environnementaux sont à considérer, qu'ils concernent la protection des ressources naturelles à l'échelle locale, ou la lutte contre le changement climatique. Dans le premier cas, le relâchement de la pression de l'élevage avicole sur l'environnement peut atténuer les problèmes : c'est le cas dans le scénario 1 où l'activité diminue, et dans le scénario 3 où l'activité se répartit sur l'ensemble du territoire. A l'inverse, une plus forte concentration de la production dans les régions à forte densité d'élevage renforcerait les préoccupations environnementales et par suite les règlementations. La déconcentration des élevages n'est pas cependant sans poser de problème car elle nécessite de nouveaux bâtiments et peut être freinée par le rejet de la population et l'éloignement des sources de matières premières pour l'alimentation animale.

Quant au changement climatique, c'est un élément de contexte important dans les scénarios 3 et 4 . Dans le premier cas, une dynamique centrée sur l'Europe ne permet pas de réduire les risques d'aggravation des problèmes environnementaux globaux, mais a des effets collatéraux positifs sur la protection des ressources locales et la consommation d'énergie. Dans le second, la coordination internationale garantit un effet plus global des mesures environnementales, mais soulève par ailleurs la question de la concurrence entre l'Homme et l'animal pour l'accès aux ressources alimentaires, ainsi que celle de la concurrence entre espèces animales pour assurer l'équilibre alimentaire de la population mondiale. La volaille dans ce contexte, est certes favorisée par un indice de consommation plutôt faible, mais elle consomme des céréales dont peuvent se passer les bovins à l'herbe, et valorise moins bien les sousproduits agricoles que le porc. Ainsi, la confrontation des différents scénarios soulève la question de la définition de la durabilité environnementale et de sa perception par le consommateur. Il importe donc de préciser quels sont les axes prioritaires pour la production avicole, entre bilan carbone, conservation de la biodiversité, préservation des paysages, lutte contre les pollutions ou contre le gaspillage, caractère naturel et/ou local, etc. La coexistence de critères multiples et de stratégies d'entreprises variées présente un risque fort de confusion pour le consommateur et une dispersion des efforts des producteurs pour améliorer la qualité de leurs produits.

\section{2 / Les leviers d'action en faveur de la filière française}

a) La structuration de la filière: exploiter au mieux les atouts français

La restructuration de l'industrie avicole est un élément majeur dans tous les scénarios. Elle peut limiter la concurrence entre entreprises de la filière française, donner plus de poids aux industriels dans la négociation avec la grande distribution, et surtout leur permettre d'affronter avec plus de force la concurrence des pays tiers. Dans tous les scénarios, cette restructuration est transnationale à l'échelle de l'Europe; elle met en concurrence les industriels mondiaux de la viande nord- et sud- américains et quelques groupes agroalimentaires européens motivés par la prise de leadership sur leur propre marché. Le scénario 3 souligne par ailleurs le rôle potentiellement déterminant des coopératives agricoles, principalement dans la mise en place de démarches de développement durable, grâce à leur ancrage territorial et à leur capacité à raisonner sur l'exploitation plutôt que sur l'atelier (complémentarité entre ateliers, entre élevage et culture, autonomie énergétique de l'exploitation...). Toutefois, pour devenir un moteur de la restructuration de la filière, les coopératives devront aussi mieux intégrer la logique de la demande, à laquelle obéissent les industriels privés.

Les nouveaux acteurs de l'aval de la filière auront intérêt à faire évoluer les contrats qui les lient aux producteurs. Ces contrats sont en effet des outils de gestion de la production qui sont maintenus dans tous les scénarios. Sous leur forme actuelle, ils semblent peu motivants pour de nouveaux éleveurs et manquent d'efficacité pour améliorer les performances et favoriser l'innovation, qu'elle réponde à des motivations technico-économiques ou à l'évolution des demandes de la société. Selon les contextes, les scénarios explorent différentes évolutions de la rémunération et des marges de manœuvres techniques des éleveurs pour améliorer les performances des élevages et revaloriser l'activité d'éleveurs dont le niveau de formation s'est considérablement élevé. Les scénarios 3 et 4 soulignent en particulier l'importance de l'introduction des exigences environnementales dans les contrats, à travers le développement de classements et de certifications d'élevage, entrainant une rémunération différentielle en fonction des performances environnementales. Outre une meilleure adéquation à la demande, cela encouragerait une dynamique d'innovation capable de maintenir une avance technique sur les concurrents (à cet égard, les contrats pourraient d'ores et déjà inciter à la conversion des élevages à la Haute Qualité Environnementale).

b) L'innovation et le développement: entre initiatives individuelles et démarches collectives

Les scénarios montrent qu'une dynamique collective est nécessaire pour aborder certains enjeux multicritères tels que le développement durable. Cependant, chaque groupe d'acteurs (positionné sur différents signes de qualité ou marques) a souvent tendance à valoriser individuellement les éléments qui lui sont le plus favorables. Si les stratégies fondées sur la recherche de compétitivité sont un bon outil d'émulation et de stimulation, elles restent néanmoins une possible source de confusion pour le consommateur (qui doit faire face au manque de lisibilité d'une diversité de signes de différenciation). En effet, les stratégies de valorisation individuelles des différents groupes d'acteurs rendent difficiles la définition d'indicateurs solides en matière environnementale, sociale ou bien-être animal. Elles limitent par ailleurs le pouvoir d'influence des acteurs de la filière sur la conception des politiques publiques. Un équilibre entre initiatives individuelles et démarches collectives doit donc être trouvé sachant que celui-ci est variable selon les contextes. Ainsi par exemple, il peut être suffisant de réduire individuellement les émissions de polluants dans les scénarios où les élevages exercent une faible pression sur l'environnement (soit parce qu'ils sont peu nombreux, soit parce qu'ils sont dispersés). Il faudra en revanche, imaginer le traitement des effluents dans un contexte de forte concentration de la production, ce qui exigera le recours à des solutions techniques nécessitant des investissements 
collectifs (valorisation des déjections comme combustible, comme fertilisant...). Par ailleurs, la rencontre de stratégies de réduction des coûts, d'une part, et de réduction des émissions de gaz à effet de serre, d'autre part, peut être un élément déclencheur de nouvelles stratégies collectives compatibles avec la poursuite de trajectoire individuelle. Ce serait le cas par exemple de la mutualisation des outils d'abattage, qui permettrait à chaque éleveur, dans la mesure du possible, de faire abattre ses animaux à proximité de son atelier. $\mathrm{A}$ une autre échelle, pour favoriser la mise en place de circuits courts, le développement d'outils industriels simples, gérés collectivement est une option à explorer.

L'équilibre entre démarches collectives et individuelles doit faire l'objet d'une réflexion des acteurs de la filière à d'autres niveaux tels que la communication vis-à-vis du grand public, la mise en place de marques, la conquête de nouveaux marchés (domestiques ou à l'export) fondée sur la différenciation, l'amélioration des caractéristiques des produits frais et ultra-frais (découpes prêt-à-cuire, cuisson aux microondes...), l'évolution des cahiers des charges des productions sous signes de qualité, etc. Toutefois, compte tenu du faible poids de l'aviculture dans l'agriculture et dans le dispositif de développement agricole, l'organisation doit être collective quand il s'agit pour la filière de s'impliquer dans les processus de conception des politiques publiques, que ce soit au niveau national ou européen. dies

c) Des politiques publiques approfon-

La filière avicole française souffre aujourd'hui d'un déficit de compétitivité en prix par rapport à plusieurs concurrents mondiaux, voire européens. Par suite, toute diminution de la protection tarifaire à l'entrée dans l'Union Européenne, sous l'effet d'un accord multilatéral de libéralisation des échanges agricoles à l'OMC ou d'accords bilatéraux de libéralisation avec les pays du Mercosur, aurait pour effets, toutes choses égales par ailleurs, d'augmenter les importations françaises de produits avicoles et de diminuer la production avicole hexagonale (cf. scénarios 1 et 3 ). De plus, le recours aux subventions à l'exportation sera vraisemblablement encore plus difficile demain qu'il ne l'est aujourd'hui.
Dans ce contexte, l'exercice de prospective montre que l'avenir de la filière avicole française, en termes de volumes de produits avicoles produits sur la base d'un minerai d'origine nationale, dépend fortement d'une politique de protection ou de différenciation par rapport à la concurrence. La protection peut être tarifaire comme un maintien de droits de douane à l'importation dans l'Union Européenne. Elle peut aussi être non tarifaire, en reconnaissant des normes plus sévères en matière d'environnement, de bien-être des animaux ou d'exigences sanitaires et en les prenant en compte à l'entrée sur le sol communautaire.

A ce titre, l'élevage avicole français gagnerait à ce que les réglementations commerciales internationales intègrent davantage les trois dimensions de l'environnement, du bien-être des animaux et de la sécurité sanitaire. Elle gagnerait aussi à un approfondissement du processus de réforme de la Politique Agricole Commune (PAC) à l'œuvre depuis deux décennies et visant à mieux valoriser les biens environnementaux, le territoire, la qualité au sens large, y compris dans sa dimension sécurité sanitaire, etc. Elle gagnerait enfin à une meilleure cohérence de la politique agricole et de la politique nutritionnelle.

d) Recherche et Développement mobilisés sur la durabilité des systèmes d'élevage et la caractérisation de la qualité des produits

Dans les années 60, les innovations dans les domaines de la génétique, de l'alimentation des animaux, de la santé animale et des bâtiments et équipements d'élevage ont constitué le principal facteur de développement de la production avicole en Europe et en Amérique du Nord (Boyd 2001). En Europe, les orientations de la recherche, sur lesquelles sont fondées les innovations, se sont élargies progressivement en lien avec l'évolution des marchés, des règlementations et de la demande sociétale en termes de qualité sanitaire (hygiène, conservation et traçabilité des produits) et de bien-être animal, de praticité des produits ou de durabilité du système de production dans ses volets économiques, sociaux et environnementaux avec notamment la question de la gestion des déchets et des pollutions, etc. Les scénarios confirment l'intérêt des orientations de la recherche actuelle et renforcent certaines orientations notamment en termes d'amélioration de la durabilité, de maîtrise de la qualité des produits et des risques sanitaires, d'organisation et d'encadrement de la profession.

La prise en considération des enjeux de la durabilité mais aussi l'importance pour la filière européenne d'afficher sa différence par rapport aux filières d'importation impliquent la conception de nouveaux systèmes d'élevage et la définition d'indicateurs de durabilité prenant en compte des composantes parfois contradictoires. Plusieurs voies d'amélioration de la durabilité des systèmes sont à explorer :

- l'identification de nouvelles matières premières permettant de réduire la concurrence avec l'alimentation humaine pour l'utilisation des céréales d'une part et la dépendance vis-à-vis du soja importé d'autre part,

- l'intégration d'une caractéristique «bien-être animal» dans la sélection des animaux d'élevage et dans les conditions d'élevage,

- l'amélioration de l'attractivité du métier d'aviculteur, qui dépend de différents facteurs, parmi lesquels l'image du métier d'aviculteur. Or celle-ci est notamment liée aux conditions de travail et aux revenus, eux-mêmes dépendant de la performance économique de la filière et du partage de la valeur ajoutée 4 .

La qualité des produits est également un domaine qui nécessite des critères d'appréciation objective, particulièrement dans un contexte de forte concurrence où la différenciation des produits est un enjeu fort pour la production domestique. Ces critères doivent être à la fois pertinents, fiables et non contestables, suffisamment faciles à mesurer, mais aussi lisibles par le consommateur. Les recherches sur les caractéristiques de la viande de poulet sont à étendre aux autres espèces avicoles et à l'ensemble des caractéristiques recherchées par les différents secteurs de la filière. Les scénarios soulignent en effet l'importance de l'ensemble des composantes (sensorielle, technologique, nutritionnelle...) de la qualité des produits et ce, quel que soit le type de commercialisation et de consommation. Toutes ces démarches doivent inclure l'étude des aspects socio-économiques de la consommation pour garantir la pleine reconnaissance des indicateurs par les consommateurs et les distributeurs. Pour atteindre des niveaux optimums, les recherches procèdent ensuite par analyse des effets des facteurs d'élevage et d'abattage, d'aptitudes génétiques particulières, des

\footnotetext{
${ }^{4}$ Ces deux derniers points sont eux-mêmes dépendants de l'amélioration de la compétitivité-prix des produits européens, qui passent en France notamment par une évolution des contrats entre les producteurs et les industriels d'amont ou d'aval. A cet égard, très peu de données sont disponibles à ce jour alors qu'une réflexion professionnelle accompagnée par des économistes semble souhaitable.
} 
process de transformation, etc. En parallèle, les stratégies permettant de garantir la sécurité d'un produit et d'améliorer sa conservation constituent des enjeux importants. La cible est également à considérer : les appliquer à l'amont de la chaîne, en particulier à la production, renforce la filière française ou européenne (comme dans le scénario 2) alors que viser la transformation peut favoriser les importations. Ces évolutions influenceront également les stratégies des distributeurs en leur permettant de garantir leurs produits et de modifier leur gestion des stocks.

Par ailleurs, il apparaît important de renforcer les recherches en sciences économiques et sociales, notamment afin d'analyser l'impact des différentes politiques publiques, dont les politiques commerciales et les règlementations, sur la production. L'exploration des systèmes de contractualisation est également une voie à développer. En effet, dans de nombreux pays, les relations de l'industrie avicole avec les éleveurs sont dominées par l'intégration (ou plus précisément par des relations de quasiintégration car les éleveurs demeurent propriétaires de leur bâtiment et parfois de leur cheptel). Cette organisation a montré son efficacité en termes d'adaptation de l'offre à la demande mais ne permet pas toujours l'optimisation des performances techniques en élevage, limitant ainsi la compétitivité globale de la filière. La comparaison des différents systèmes pratiqués en Europe permettrait de dégager les atouts et handicaps de différentes formes d'organisation de filières et de les faire évoluer.

Enfin, le développement des approches sociologiques du métier de producteur avicole et de son attractivité permettrait de comprendre l'importance des différents éléments, économiques ou non, qui influent sur les choix d'élevage et ainsi d'orienter les travaux sur l'amélioration des conditions de vie des producteurs. En parallèle, il importe de mettre en place un système permettant la reconnaissance de la qualité du produit et par suite du travail de l'éleveur.

L'ensemble de ces perspectives implique des approches pluridisciplinaires pour pouvoir appréhender les différents éléments de réponse et s'appuyer sur des éléments d'appréciation objectifs de systèmes. Il faudra également transférer les connaissances scientifiques aux acteurs de la filière pour permettre la mise en place de la démarche et par suite les évolutions de la filière.

\section{Conclusion}

En termes de production, si aucun scénario n'envisage un retour aux fortes valeurs des années 90, tous, toutefois, mettent en évidence des évolutions en rupture avec la chute de production observée depuis une dizaine d'années. Entre les deux extrêmes, la filière dispose de marges de manœuvre relevant des stratégies d'acteurs, des politiques publiques ou des orientations de la recherche. Lorsqu'il s'agit de contribuer à la définition ou à la mise en place de politiques publiques et de réglementations, ou de collaborer à des projets de recherche, la coordination des acteurs est un élément clé pour éviter la dispersion des efforts de chacun. La forte concurrence étrangère sur les prix ne fait que renforcer cette nécessité dès lors qu'il s'agit pour la filière de se protéger ou de se différencier par rapport à ses concurrents américains ou asiatiques.

Par ailleurs, la réflexion soulève l'importance de la durabilité de l'aviculture dans un contexte où l'on s'interroge sur la manière de nourrir une population croissante sans compromettre le renouvellement des ressources naturelles. Confrontée à des enjeux sanitaires ou de bien-être animal, la filière a fort intérêt à développer des synergies entre ces aspects et les dimensions économiques, sociales et environnementales de la durabilité. Mais concilier les enjeux environnementaux et les exigences des consommateurs constitue en soi un défi qui ne peut parfois être relevé que par l'évolution des systèmes de production, accompagné d'innovations organisationnelles et technologiques. La difficulté augmente quand, pour satisfaire la demande de proximité, se pose la question du rééquilibrage territorial et de son accueil par la population. C'est le cas en particulier quand il s'agit de promouvoir des systèmes de production favorisant la complémentarité entre céréales et élevages ou d'approvisionner via des circuits courts des bassins de consommation éloignés des zones de production de céréales et/ou des ports d'importation des matières premières pour l'alimentation des animaux.

Enfin, envisager les solutions qui permettront de concilier les différents objectifs de la production ne va pas sans considérer qu'à l'interface entre le lien à la nature et le bien-être animal, le rejet de l'artificialisation de l'élevage, est un élément fort de la perception de la production par la population et qu'il constitue aujourd'hui un point faible de la production avicole. Le consommateur sera-t-il prêt à accepter une forte rationalisation environnementale de l'élevage, dans des conditions fortement artificialisées ? Ou, à l'opposé, préfèrera-t-il orienter ses choix vers des produits de type bio plein air utilisant des animaux à croissance lente même si ceux-ci sont pénalisés par un plus fort bilan carbone 5 ? Ce type de dilemme souligne d'une part l'intérêt, voire la nécessité, pour les chercheurs de mettre en œuvre des approches systémiques et pluridisciplinaires, et d'autre part pour les acteurs de la filière, d'être proactifs dans le cadre d'une réflexion collective.

\section{Remerciements}

Les auteurs tiennent à exprimer leur reconnaissance à l'ensemble du groupe de travail pour leur apport déterminant à cette prospective. Ils remercient les relecteurs.

\footnotetext{
${ }^{5}$ La garantie d'une qualité organoleptique supérieure exige un abattage tardif et des génotypes différents, pénalisant pour le bilan carbone dans la mesure où la consommation d'aliments est supérieure à celle d'un animal élevé sur une durée plus courte.
} 


\title{
Références
}

Agreste, 2001. Les principaux résultats du recensement agricole 2000. Ministère de l'Agriculture et de la Pêche, Cahiers Agreste, 3-4, Décembre 2001, 90p

Agreste Chiffres et Données, 2006. Enquête aviculture 2004. Ministère de 1'Agriculture et de la Pêche, Série Agriculture, 176, 86p.

Agreste Conjoncture, 2008. Regain de la consommation de viande blanche en 2007. Ministère de l'Agriculture et de la Pêche, Synthèses, 29, 5p.

And-I, 2008. Panoramas financiers sectoriels, industrie de la volaille, synthèse des résultats 2007-2008, Paris, France, 95p.

Boyd W., 2001. Making meat: Science, technology and american poultry production. Technology and Culture, 42, 631-664.
Combris P., 1997. La consommation de produits animaux en France : tendances et perspectives d'évolution. Viandes Prod. Carn., 18, 19-36.

De Jouvenel H., 2004. Invitation à la prospective. Editions Futuribles, collection Perspectives, Paris, France, 88p.

Jez C., Beaumont C., Magdelaine P., Paillard S., 2009. La filière avicole française à l'horizon 2025. INRA-ITAVI, Paris, France, 89p.

Lavergne P., 2003. Les contrats dans l'aviculture : quels apports de l'économie ? 5 èmes Journ. Rech. Avicole, 26 et 27 Mars, Tours, France, 4p.

Magdelaine P., 2008. La situation des filières avicoles françaises. Rapport réalisé dans le cadre du projet AVITER-ITAVI, Paris, France, $75 \mathrm{p}$.
Magdelaine P., Spiess M.P., Valceschini E., 2008. Poultry meat consumption trends in Europe. World's Poult. Sci. J., 64, 53-64.

OECD, 2006. The implications of changing market structures in the poultry industry. AGR/CA/APM/CFS/MD/RD(2006)7.

Office de l'élevage, 2008. Le marché des produits laitiers, carnés et avicoles en 2008. Paris, France, 520p.

Trégaro Y., Vallin B., 2009. Les filières avicoles européennes face à la mondialisation des échanges. 8èmes Journ. Rech. Avicole, 25 et 26 mars, St-Malo, France, 7p.

Van Horne P., 2009. Coûts de production des poulets de chair dans différents pays du monde: étude comparative et perspectives.

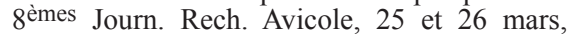
St-Malo, France, 4p.

\section{Résumé}

Depuis une dizaine d'années, la filière avicole française doit faire face à la concurrence de nouveaux pays producteurs plus compétitifs ; elle doit également répondre à de nouvelles exigences de la société vis-à-vis des modes de production en termes de bien-être animal, de sécurité sanitaire et de protection de l'environnement. Ainsi, si depuis 10 ans, la production mondiale a bénéficié d'une croissance de près de $4 \%$ par an, la production française a baissé au rythme de $2,3 \%$ par an. Les acteurs économiques du secteur et les acteurs publics s'interrogent sur cette évolution et, surtout, sur les perspectives d'avenir de l'aviculture. Pour éclairer leurs choix stratégiques, un institut technique (ITAVI) et un institut de recherche (INRA) se sont mobilisés autour d'un exercice de prospective. Fondé sur les réflexions d'un groupe de travail composé d'experts des filières avicoles et sur des auditions d'acteurs, l'exercice a permis de construire quatre scénarios à partir des évolutions du contexte politique et réglementaire, des comportements de consommation et des stratégies des acteurs de la filière. L'exploration de différents futurs possibles à l'horizon 2025 a permis d'identifier des leviers d'action relevant des stratégies d'acteurs, des politiques publiques et des orientations de la recherche. Si aucun scénario n'envisage un fort développement de la production à l'horizon 2025, tous, cependant, montrent l'intérêt d'une recherche multidisciplinaire pour caractériser la durabilité et la qualité de la production, et les rendre conciliables avec une meilleure compétitivité.

\begin{abstract}
French poultry in 2025. INRA-ITAVI foresight

For about ten years, the French poultry sector has faced both competition from new poultry-exporting countries and growing societal concerns about animal welfare, food safety, and environmental impact of production systems. As such, while world poultry-meat production increased nearly $4 \%$ per year during that period, it decreased by $2.3 \%$ per year in France, making poultry farmers, public and other private stakeholders worried about the future of poultry farming. To help French stakeholders design their strategies for the future, a technical institute (ITAVI) and a research institute (INRA) conducted a scenario-building exercise. Based on the interviews of stakeholders and an 18 month series of discussions with a Panel of poultry experts, four future scenarios were developed. They go beyond simply extending current trends by taking into account uncertainties such as potential shifts in European policies and regulations, consumer attitudes, and stakeholder strategies. Levers of action were identified by the exploration of different possible futures for poultry in $\mathbf{2 0 2 5}$. These strategic options concern the stakeholders' strategies, public policy and research. Although no scenario envisages strong production growth by 2025 , all, however, underline the importance of multidisciplinary research to characterize the quality and sustainability of production, and, in turn, to improve competitiveness.
\end{abstract}

JEZ C., BEAUMONT C., MAGDELAINE P., 2010. La filière avicole française à l'horizon 2025. Une prospective INRAITAVI. Inra Prod. Anim., 23, 379-390. 
